\title{
Progress of Divertor Study on DEMO Design*
}

\author{
Kazuo HOSHINO, Nobuyuki ASAKURA ${ }^{1)}$, Shinsuke TOKUNAGA ${ }^{1)}$, Katsuhiro SHIMIZU, \\ Yuki HOMMA ${ }^{1)}$, Youji SOMEYA ${ }^{1)}$, Hiroyasu $\mathrm{UTOH}^{1)}$, Yoshiteru SAKAMOTO ${ }^{1)}$, Kenji TOBITA ${ }^{1)}$ \\ and The Joint Special Design Team for fusion DEMO \\ National Institutes for Quantum and Radiological Science and Technology, Naka, Ibaraki 311-0195, Japan \\ ${ }^{1)}$ National Institutes for Quantum and Radiological Science and Technology, Rokkasho, Aomori 223-2189, Japan
}

(Received 4 July 2016 / Accepted 3 April 2017)

Recent progress of the physics and engineering design study for the $8 \mathrm{~m}$-sized DEMO is reported. Parametric study for the divertor of the compact DEMO (a machine size $\sim 5.5 \mathrm{~m}$ ) by using the SONIC code shows that the target heat load less than $10 \mathrm{MW} / \mathrm{m}^{2}$ around the fusion power of $\sim 1.5 \mathrm{GW}$ and the impurity radiation fraction of more than $80 \%$. In the $8 \mathrm{~m}$ sized DEMO with these parameters, the partial detachment is obtained at the outer divertor, even in the low SOL density, due to the large impurity radiation in the SOL and divertor region. The SONIC simulation shows the peak of the target heat load is $7 \mathrm{MW} / \mathrm{m}^{2}$. However, the peak of the ion temperature at the target is considerably high, which causes significant erosion of the target. The divertor power handling and decrease in the ion temperature have to be proceeded by the scenario development of the divertor plasma operation as well as the core plasma design and the engineering design. In the engineering study side, the tungsten monoblock target with the water cooling and the Cu-alloy cooling tube is designed. The MCNP-5 neutronics analysis shows applicability of the Cu-alloy cooling tube for the divertor unit on the high heat flux region. Also the divertor cassette with a heat removability of peak heat load of $10 \mathrm{MW} / \mathrm{m}^{2}$ is studied. The heat transport analysis shows the maximum temperature of $1021^{\circ} \mathrm{C}$ at the tungsten surface and $331^{\circ} \mathrm{C}$ at the Cu-alloy pipe, which are acceptable level for mechanical toughness and thermal fatigue.

(c) 2017 The Japan Society of Plasma Science and Nuclear Fusion Research

Keywords: fusion DEMO reactor, divertor, physics design, engineering design

DOI: $10.1585 /$ prr.12.1405023

\section{Introduction}

Conceptual design study of a fusion DEMO reactor in japan is being progressed under the framework of the ITER broader approach and the Joint Special Design Team for fusion DEMO. One of the crucial issues in the DEMO design is the huge power handling in the SOL/divertor region. While the divertor has important roles, such as control of the power and fuel particle exhausted from the core region, exhaust of the $\mathrm{He}$ ash, control of the impurity flow, and so on, the huge power exhausted from the core, which is several times larger than that of ITER, is concentrated on the divertor. Therefore, reduction of the heat load on the divertor target and advancement of the target heat removal are of considerable significance.

The desirable heat load on the DEMO divertor target is less than or comparable to the ITER design of $10 \mathrm{MW} / \mathrm{m}^{2}$ because the available material is restricted by the strong neutron irradiation environment. The heat removal capability is evaluated to be below $5-8 \mathrm{MW} / \mathrm{m}^{2}$ for a monoblock target with tungsten and reduced activation ferritic martensitic (RAFM) steel as the structure and the watercooling pipe materials [1].

author's e-mail: hoshino.kazuo@qst.go.jp

*) This article is based on the invited talk at the 32nd JSPF Annual Meeting (2015, Nagoya).
The power handling scenario for a $3 \mathrm{GW}$ class fusion reaction with an ITER-sized plasma $(\sim 5.5 \mathrm{~m})$, SlimCS $[1,2]$, has been investigated [3-5]. The primary technique for reduction of the divertor heat load to such a desirable level is enhancement of the radiation loss by impurity gas seeding. The SONIC simulation showed that the target heat load $q_{\text {target }}$ was $16 \mathrm{MW} / \mathrm{m}^{2}$ even in the case where more than $90 \%$ of the exhausted power from the core plasma $\left(P_{\text {out }}\right)$ was radiated by the argon (Ar) impurity gas seeding. The effects of the seeded impurity species (neon or krypton) and the divertor geometry on the divertor performance have been also investigated. Due to such effects, $q_{\text {target }}$ could be decreased but it was still larger than the heat removal capability of the divertor target. The impact of the divertor geometry has been also investigated [6]. Although the long divertor leg (distance from the $\mathrm{X}$-point to the target) has advantage on the formation of the detached divertor plasma and reduction of the target heat load, $q_{\text {target }}$ was still about $10 \mathrm{MW} / \mathrm{m}^{2}$. As for another divertor geometry, investigation of an advanced divertor concept, such as short super-X divertor has been also progressed [7].

In 2014, a new JA DEMO concept has been proposed [8]. The major parameters are as follows: $P_{\text {fus }} \sim 1.5 \mathrm{GW}$, the major radius of $8.5 \mathrm{~m}$, the minor radius of $2.4 \mathrm{~m}$, the plasma current of $12.3 \mathrm{MA}$, and the toroidal magnetic field 

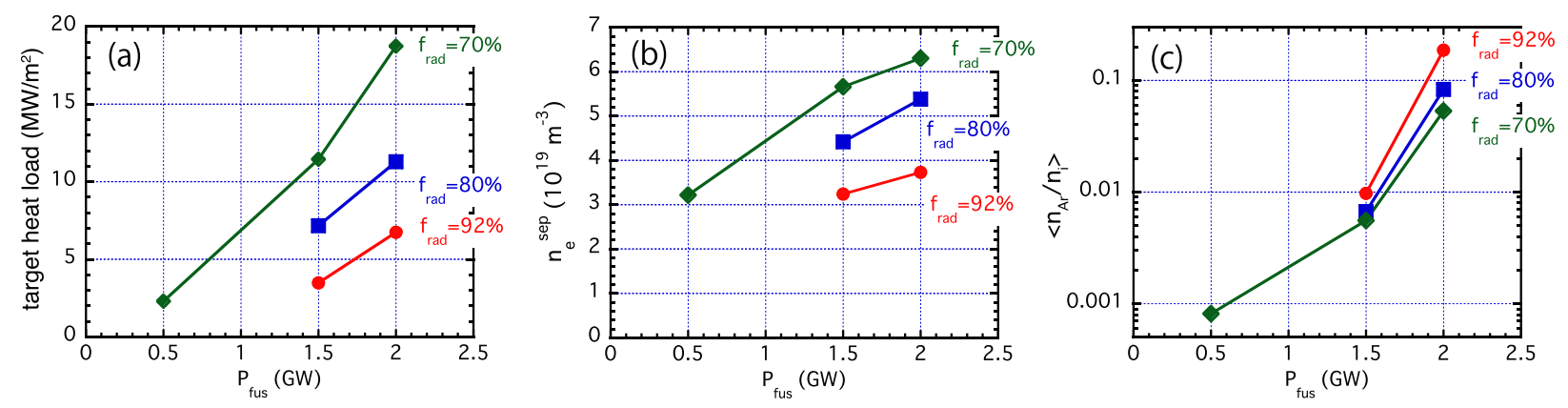

Fig. 1 The dependency of (a) the peak heat load at the outer target (b) the electron density at the outer mid-plane separatrix $n_{\mathrm{e}}^{\text {sep }}$ and (c) the impurity concentration $\left\langle n_{\mathrm{Ar}} / n_{\mathrm{i}}\right\rangle$ averaged in the edge region $(0.95<r / a<1)$ on the fusion power $P_{\text {fus }}$ and the impurity radiation fraction $f_{\text {rad. }}$.

of 5.9 T. In comparison with the previous SlimCS concept, the major radius was increased to ensure sufficient size of a center solenoid coil for the current ramp up. Also, the fusion power was decreased for safety, divertor power handling, blanket design and so on. In this paper, recent progress of the physics and engineering divertor design study for the $8 \mathrm{~m}$-sized DEMO, such as JA model 2014, is presented.

\section{Physics Design Study}

\subsection{Numerical model}

A suite of integrated divertor codes SONIC $[9,10]$ is applied to the physics design study of the DEMO divertor plasma. The SONIC suite consists of the 2-D plasma fluid code (SOLDOR), the neutral Monte-Carlo kinetic code (NEUT2D) and the impurity Monte-Carlo kinetic code (IMPMC). The transport of bulk ion is described in the fluid approximation by the SOLDOR code, while neutral particles and impurities are treated by the NEUT2D and IMPMC codes, respectively.

Reproduction of the detachment is one of the crucial issues for the divertor plasma modeling of future fusion devices, such as DEMO, ITER, JT-60SA, etc. Recently, the SONIC has been improved to include: (1) contribution from the impurities to the electron density, (2) the ion heat flux limiter, (3) smoothing source terms near the target to reduce the Monte-Carlo noise/perturbation, and (4) time averaging of the impurity Monte-Carlo result to reduce the large oscillation. The improved SONIC code can qualitatively reproduce the characteristics of the JT-60U detached divertor plasma [11].

In the DEMO divertor, the complete detachment is required for reduction of the target heat load as well as suppression of the target erosion. The neutral density in the divertor and sub-divertor region becomes higher than the existing tokamak devices. Therefore, improvement of the neutral transport model is more important. Implementation of the photon trapping effects [12] and the neutral-neutral elastic collision [13] are now under way. The former en- hances the ionization due to the photo excitation. The latter increases the neutral density close to the divertor target and enhances the pumping flux. These would affect the detachment characteristics in the DEMO divertor analysis.

\subsection{Analysis of the fusion power and the im- purity radiation}

Impacts of the fusion power and the impurity radiation on the divertor power handling is analyzed on the SlimCS divertor geometry [14], i.e., 5.5 m-sized DEMO. In the SONIC simulation, the Ar impurity gas is injected to enhance the impurity radiation and its seeding rate is adjusted to sustain the impurity radiation power set out as an input parameter. The SONIC results are summarized in Fig. 1.

Figure 1 (a) shows the peak heat load on the outer target as a function of the fusion power, $P_{\text {fus }}$, and the fraction of the impurity radiation power on the power exhausted from the core, $f_{\text {rad. }}$. Here, the target heat load includes the heat load due to the plasma heat transport, the surface recombination of the ion particle, the impurity radiation near the target and the neutral transport. The target heat load decreases with decreasing in $P_{\text {fus }}$ or increasing in $f_{\text {rad }}$. The SONIC simulation shows the target heat load less than $10 \mathrm{MW} / \mathrm{m}^{2}$ at $P_{\text {fus }}=1.5 \mathrm{GW}$ and $f_{\text {rad }} \geq 80 \%$.

As shown in Fig. 1(b), the mid-plane density $n_{\mathrm{e}}^{\text {sep }}$ tends to increase with decreasing in $f_{\text {rad }}$ or increasing in $P_{\text {fus }}$. This trend can be understood as the following process of particle balance: first, target recycling becomes lower due to them. The neutral pressure at the divertor and sub-divertor region and the resultant pumping flux are decreased. As a result, the density in the system, i.e., $n_{\mathrm{e}}^{\text {sep }}$, increases. With increasing in $n_{\mathrm{e}}^{\text {sep }}$, the divertor recycling is enhanced and the pumping flux recovers. Consequently, $n_{\mathrm{e}}^{\text {sep }}$ increases until the pumping flux balances with the influx into the system. Although the low impurity concentration in the core region is preferable for the core plasma design, the resultant increase in $n_{\mathrm{e}}^{\text {sep }}$ due to the low $f_{\text {rad }}$ may become issue in consistency with the core plasma design.

The Ar impurity concentration $\left\langle n_{\mathrm{Ar}} / n_{\mathrm{i}}\right\rangle$ averaged in 
the edge region $(0.95<r / a<1)$ is plotted in Fig. 1 (c). In the $P_{\text {fus }}=2 \mathrm{GW}$ case, the impurity concentration is significantly high to achieve high $f_{\text {rad }}=70-92 \%$. Such high concentration may be inconsistent with the core plasma design. With decreasing $P_{\text {fus }}$ to less than $1.5 \mathrm{GW},\left\langle n_{\mathrm{Ar}} / n_{\mathrm{i}}\right\rangle$ decreases to less than 0.01 which perhaps acceptable for the core plasma design. The fusion power of $\sim 1.5 \mathrm{GW}$ seems to be preferable for the conventional divertor concept in the viewpoint of the impurity concentration as well as the target heat load.

\subsection{Divertor plasma analysis for the $8 \mathrm{~m}$ - sized DEMO}

Based on above result, the divertor power handling for the $8 \mathrm{~m}$-sized DEMO with $P_{\text {fus }}=1.5 \mathrm{GW}$ and $f_{\text {rad }}=80 \%$ is investigated. Advantages of the larger sized DEMO is larger wetted area and the resultant lower target heat load. Therefore, the target heat load is expected to decrease to less than $5 \mathrm{MW} / \mathrm{m}^{2}$. However, the core electron density decreases compared with the small sized DEMO because of the lower Greenwald density $n_{\mathrm{GW}}$ due to the low plasma current [8]. In the JA model 2014, the core density, $n_{\text {core }}$, is $\sim 6.6 \times 10^{19} \mathrm{~m}^{-3}\left(n_{\text {core }} / n_{\mathrm{GW}} \sim 1.2\right)$ while it is $\sim 1.15 \times 10^{20} \mathrm{~m}^{-3}$ in the SlimCS $\left(n_{\text {core }} / n_{\mathrm{GW}} \sim 1.0\right)$ [1]. Therefore, $n_{\mathrm{e}}^{\mathrm{sep}}$ is also decreased in the $8 \mathrm{~m}$-sized DEMO. Under the same $P_{\text {fus }}$ and $f_{\text {rad }}$, the lower $n_{\mathrm{e}}^{\text {sep }}$ is disadvantage for formation of the detachment and reduction of the divertor heat load. For example, $n_{\mathrm{e}}^{\mathrm{sep}}$ of more than $\sim 3 \times 10^{19} \mathrm{~m}^{-3}$ was required in the partial detachment (the target heat load $\leq 10 \mathrm{MW} / \mathrm{m}^{2}$ ) for ITER divertor [15]. In this study, the divertor heat load is analyzed from the low $n_{\mathrm{e}}^{\text {sep }}$ case, i.e., no fuel gas puffing for the SOL/divertor plasma.

Figure 2 shows the numerical grid for the SONIC simulation. The simulation condition is as follows: the particle flux and the energy flux across the core interface boundary $(r / a \sim 0.95)$ is $1.0 \times 10^{22} \mathrm{~s}^{-1}$ and $250 \mathrm{MW}$, respectively. The pumping speed is set to be $63 \mathrm{~m}^{3} / \mathrm{s}$. The radial diffusion coefficients for the particle and energy are $0.3 \mathrm{~m}^{2} / \mathrm{s}$

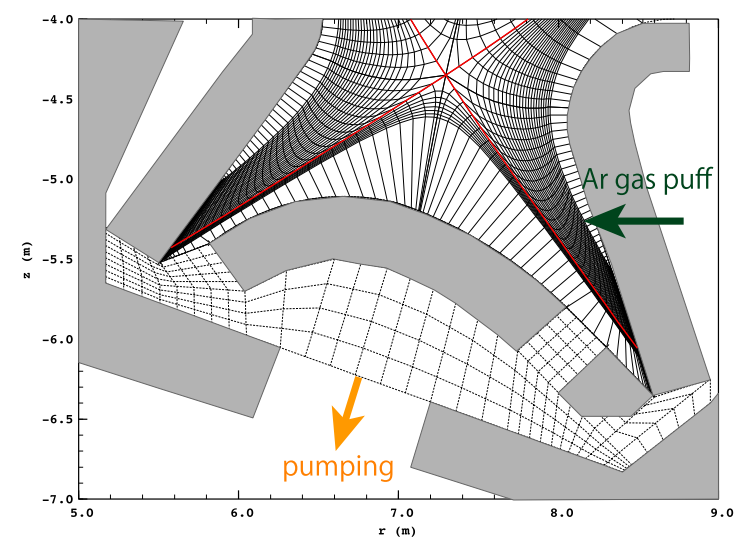

Fig. 2 The divertor geometry and the numerical grid for the SONIC simulation. and $1.0 \mathrm{~m}^{2} / \mathrm{s}$, respectively, which are the same coefficients in the ITER simulation [16]. In order to enhance the impurity radiation, the Ar impurity gas is injected near the outer divertor. The gas puff rate of the Ar impurity is adjusted to achieve $f_{\text {rad }}=0.8$. In order to reduce the calculation time for the impurity particle trace, the backflow model [17] is adopted.

Figure 3 (a) shows the radial profiles of $n_{\mathrm{e}}$, the electron temperature $T_{\mathrm{e}}$ and the ion temperature $T_{\mathrm{i}}$ at the outer mid-plane. The electron density at the outer mid-plane separatrix is $\sim 1.4 \times 10^{19} \mathrm{~m}^{-3}$, which is much lower than results of the previous section. Due to the low SOL density and large $f_{\text {rad }}$, the impurity concentration is an order of percent in the SOL region.

At the outer target, as shown in Fig. 3 (b), the detachment region $\left(T_{\mathrm{e}}, T_{\mathrm{i}}<2 \mathrm{eV}\right)$ can be seen around the strike point $(\leq 12 \mathrm{~cm})$ even in the low $n_{\text {sep }}$, due to the large $f_{\text {rad }}$ of $80 \%$. However, at the outer region, $T_{\mathrm{i}}$ exceeds $300 \mathrm{eV}$ due to the low $n_{\mathrm{e}}^{\text {sep }}$ and the resultant low recycling. Such high $T_{\mathrm{i}}$ leads to significant erosion of the target. As shown in Fig. 4, the target heat load is less than $7 \mathrm{MW} / \mathrm{m}^{2}$, which can be handled by the tungsten mono-block target with the $\mathrm{Cu}$-alloy cooling tube. The contribution to the target heat load is almost plasma heat load. Other component due to the surface recombination, the impurity radiation and the
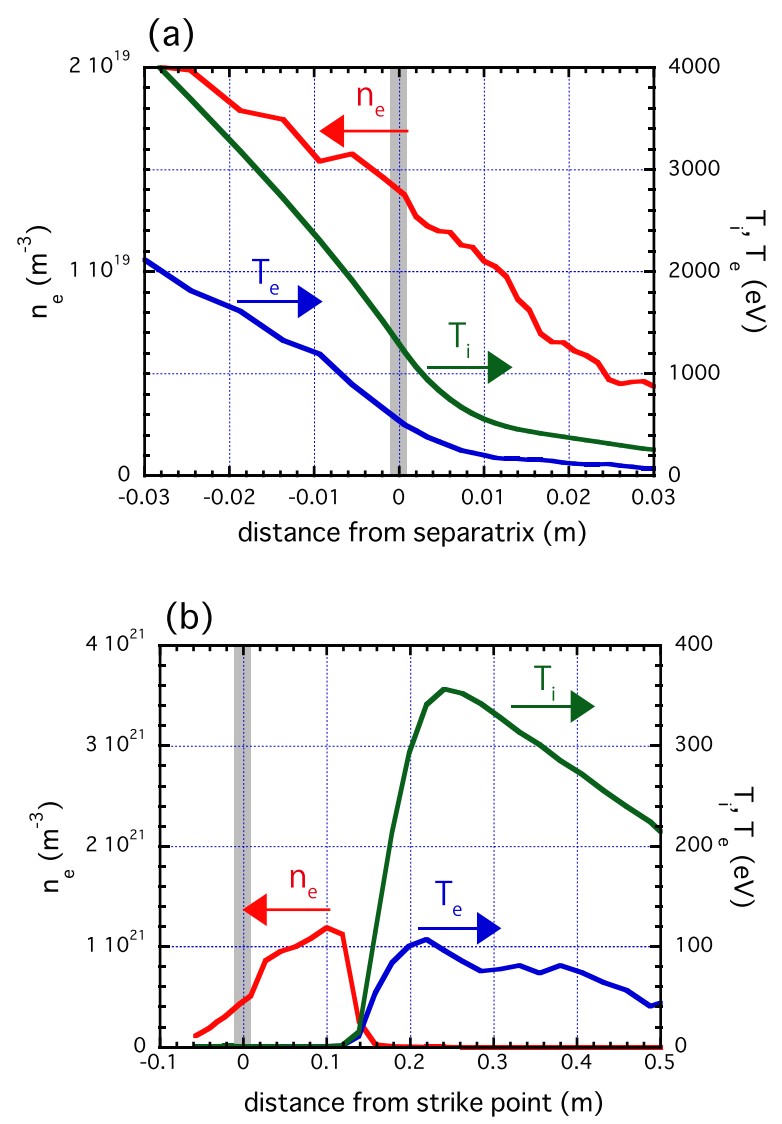

Fig. 3 The radial profile of $n_{\mathrm{e}}, T_{\mathrm{e}}$ and $T_{\mathrm{i}}$ at (a) the outer midplane and (b) the outer divertor target. 


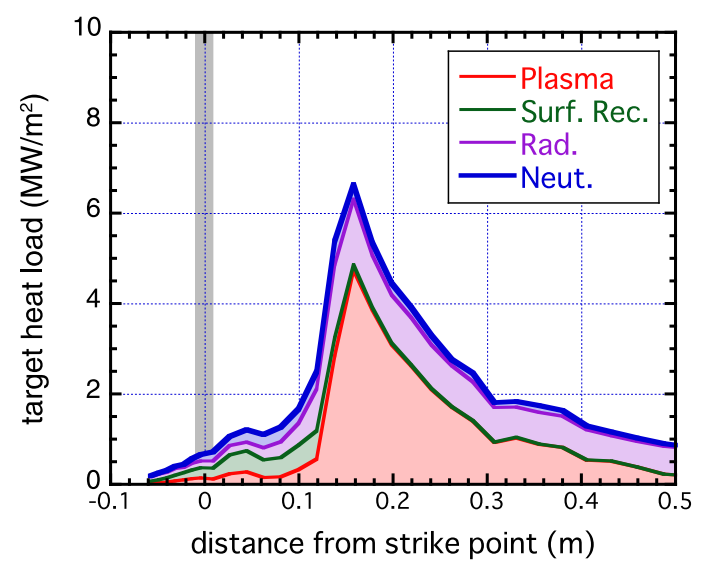

Fig. 4 The target heat load at the outer divertor target. The heat load due to the plasma heat transport, the surface recombination of the ion particles, the impurity radiation and the neutral transport are stacked.

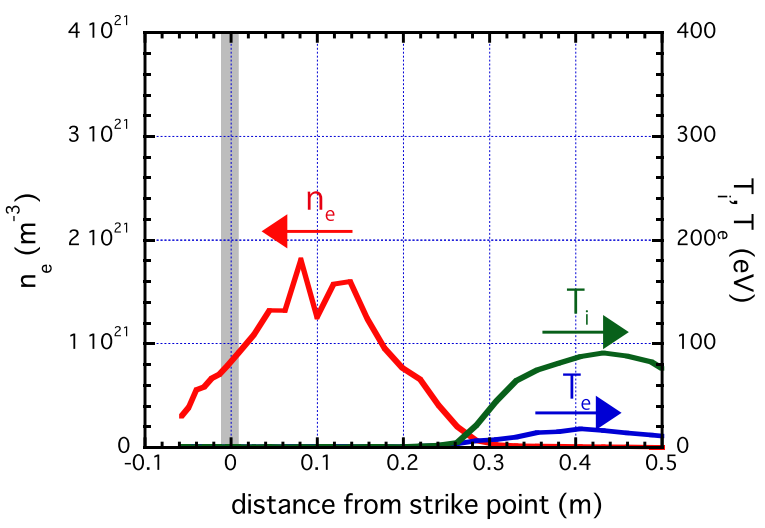

Fig. 5 The radial profile of $n_{\mathrm{e}}, T_{\mathrm{e}}$ and $T_{\mathrm{i}}$ at the outer divertor target for the gas puffing case.

neutral transport is not so large because the divertor plasma is low recycling regime at the outer region.

In order to reduce peak $T_{\mathrm{i}}$ and the resultant significant target erosion, the injection of the fuel gas with $100 \mathrm{~Pa} \mathrm{~m}^{3} / \mathrm{s}$ from the outer mid-plane is attempted. The recycling at the outer divertor target is enhanced and the peak $T_{\mathrm{i}}$ decreases to less than $100 \mathrm{eV}$ as shown in Fig. 5. In this case, $n_{\mathrm{e}}^{\text {sep }}$ increases to $3 \times 10^{19} \mathrm{~m}^{-3}$. Although further low $T_{\mathrm{i}}$ would be required to save the target erosion, further high $n_{\mathrm{e}}^{\text {sep }}$ may be inconsistent with the core plasma design $\left(n_{\text {core }} \sim 6.6 \times 10^{19} \mathrm{~m}^{-3}\right)$. Expansion of the detachment region and decrease in $T_{\mathrm{i}}$ by the impurity gas puffing, optimization of the divertor geometry etc. is necessary. In addition, development of the core plasma design, such as a scenario of the high impurity radiation in the core region, etc. is indispensable.

\section{Engineering Design Study}

Basic concept of the divertor target is water-cooled tungsten monoblock, similar to ITER divertor design. As

\subsection{GW-Fusion power}
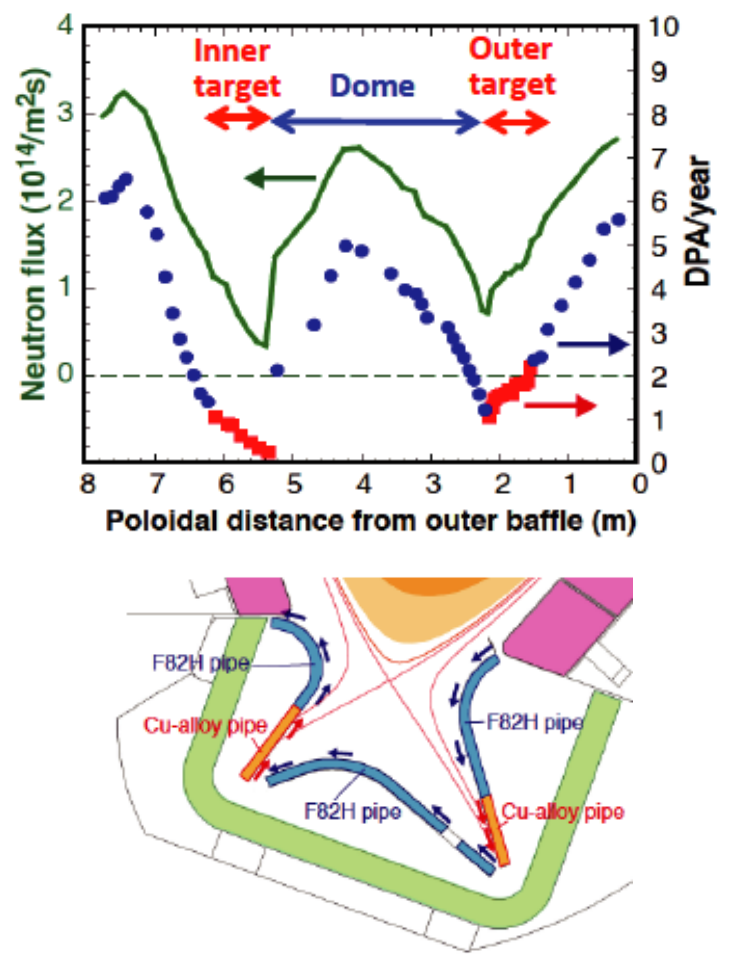

Fig. 6 Distribution of the neutron flux and DPA along the divertor target and dome.

mentioned in Sec. 1, the available material for the divertor is restricted by the strong neutron irradiation environment, especially in the compact and high performance DEMO such as SlimCS. Therefore, RAFM steel cooling-pipe was designed for SlimCS. In this target design, the heat removal capability was evaluated below $5-8 \mathrm{MW} / \mathrm{m}^{2}$. In JA model 2014, the machine size increased and the fusion power decreased, and therefore the neutron irradiation environment is alleviative. The neutronics analysis and conceptual design of the divertor target using $\mathrm{Cu}$-alloy cooling-pipe is progressed.

\subsection{Neutronics analysis}

Neutronics analysis using MCNP-5 neutron transport code and FENDL-2.1 nuclear database is carried out. The neutron source is given based on the core plasma profile for $P_{\text {fus }}=1.5 \mathrm{GW}$. In the analysis, the water cooling pipe of $\mathrm{Cu}$-alloy $(\mathrm{CuCrZr})$ is applied for the high heat flux region (the plasma wetted area), while RAFM steel (F82H) pipe is used for other regions.

Distributions of the neutron flux and the displacements per atom (DPA) rate of the water cooling pipe on the divertor are shown in Fig. 6. The unit with the $\mathrm{Cu}$-alloy (the inner and outer targets) and RAFM (the dome and baffles) cooling-pipe are shown by square and circle symbols, respectively. DPA rate of the high heat flux region $(\mathrm{Cu}-$ alloy pipe) is $0.2-2$. In the viewpoints of the hardening 


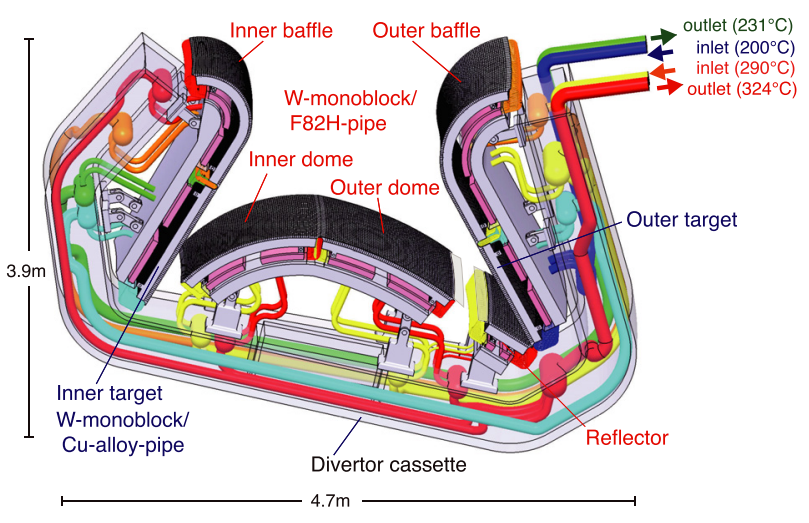

Fig. 7 Divertor cassette and the cooling water route.

and swelling under neutron irradiation, $\mathrm{Cu}$-alloy is available for a few DPA $[18,19]$. Therefore, the Cu-alloy cooling pipe can be applied for the divertor unit on the high heat flux region, while the replacement of the $\mathrm{Cu}$-alloy cooling unit is expected every $1-2$ years. The DPA rate at the baffles and dome is increased up to 5-7, and therefore RAFM cooling pipe is necessary for the lower heat load condition $\left(q_{\text {target }}<4-5 \mathrm{MW} / \mathrm{m}^{2}\right)$.

\subsection{Divertor design and arrangement of the cooling water route}

The divertor cassette with two different cooling route is designed, as shown in Fig. 7. The W-monoblock with the $\mathrm{Cu}$-alloy cooling pipe is arranged on the high heat load region of $1.0 \mathrm{~m}$ and $0.8 \mathrm{~m}$ at the inner and outer divertor target, respectively. Condition of the cooling water is assumed $200^{\circ} \mathrm{C}$ and $5 \mathrm{MPa}$ at the inlet. At the lower heat load region of the target, the baffles and the dome, the RAFM pipe with $290^{\circ} \mathrm{C}$ and $15 \mathrm{MPa}$ at the inlet is used due to the strong neutron flux.

The heat removal capability of the divertor cassette and required coolant flow speed are evaluated. Distribution of the total heat load is given by the SONIC result. The peak heat load at the outer target is increased to $10 \mathrm{MW} / \mathrm{m}^{2}$ as maximum of high heat load region. The neutron flux calculated in Sec. 3.1 is also considered. A cooling route for the $\mathrm{Cu}$-alloy pipe and 4 cooling routes for the RAFM pipe are designed.

The route of the $\mathrm{Cu}$-alloy pipe passes in the high heat load region from the outer target to the inner target. The flow velocity is $9.7 \mathrm{~m} / \mathrm{s}$ and $13.7 \mathrm{~m} / \mathrm{s}$ for the outer and inner target, respectively. The water temperature increases from $200^{\circ} \mathrm{C}$ at the inlet to $250^{\circ} \mathrm{C}$ at the outlet.

The flow velocity of the RAFM-pipe route for cooling of the outer and inner baffle is $4.0 \mathrm{~m} / \mathrm{s}$ and $5.4 \mathrm{~m} / \mathrm{s}$, respectively, to keep the water temperature below $325^{\circ} \mathrm{C}$ at the outlet. The flow velocity of other 3 route of the RAFM pipe to the inner and outer domes and outer reflector is 4.5 , 4.0 and $0.5 \mathrm{~m} / \mathrm{s}$, respectively.

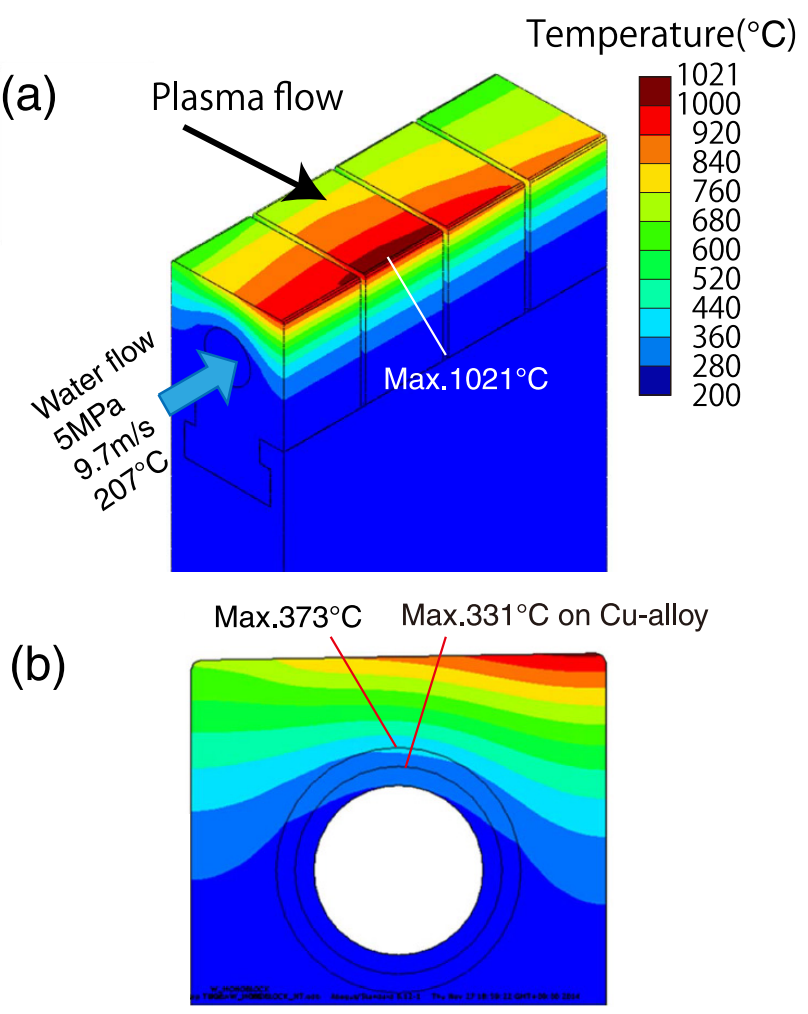

Fig. 8 Distribution of the surface temperature for (a) four monoblocks near the separatrix and (b) the cross-section of the monoblock.

\subsection{Heat transport analysis}

Based on above analyses, the tungsten monoblock with the $\mathrm{Cu}$-alloy cooling tube is designed. Size of one monoblock is $22 \mathrm{~mm} \times 15 \mathrm{~mm} \times 22 \mathrm{~mm}$. Bore diameter and thickness of a Cu-alloy cooling pipe is $9 \mathrm{~mm}$ and $1 \mathrm{~mm}$, respectively. Interlayer between tungsten and $\mathrm{Cu}-$ alloy pipe is $\mathrm{Cu}-\mathrm{OFHC}$ with $1 \mathrm{~mm}$ thickness. The support structure is $\mathrm{F} 82 \mathrm{H}$. The tile surface is tilted by $1^{\circ}$ in the toroidal direction in order to avoid melting of the leading edge, and the minimum distance between the tile surface and the interlayer is $5 \mathrm{~mm}$.

Heat transport analysis of the monoblock is performed by the ABAQUS code with three-dimensional modeling. Four monoblocks and cooling pipe with coolant flow velocity of $9.7 \mathrm{~m} / \mathrm{s}$ and inlet temperature of $207^{\circ} \mathrm{C}$ are modeled. The heat load is as same as that in Sec.3.2. Distributions of the temperature on the surface and cross-section of the monoblock are shown in Figs. 8 (a) and (b), respectively. The maximum temperature of $1021^{\circ} \mathrm{C}$ appears near the downstream side edge. The value is lower than critical temperature of recrystallization above $1200^{\circ} \mathrm{C}$. At the side surface, $\mathrm{Cu}$-OFHC interlayer and the $\mathrm{Cu}$-alloy pipe has the maximum temperature of $372^{\circ} \mathrm{C}$ and $331^{\circ} \mathrm{C}$, respectively. Mechanical toughness of the cooling pipe is maintained and these values are acceptable against thermal fatigue. 


\section{Summary}

The physics and engineering design study of the divertor has been progressed for the $8 \mathrm{~m}$-sized DEMO.

The divertor plasma performance was analyzed by the SONIC code. Parametric study on the SlimCS divertor geometry showed that the target heat load less than $10 \mathrm{MW} / \mathrm{m}^{2}$ around the fusion power of $1.5 \mathrm{GW}$ and the impurity radiation fraction of more than $80 \%$. Based on the parametric study, the divertor power handling for the $8 \mathrm{~m}$-sized DEMO with the fusion power of $1.5 \mathrm{GW}$ and the impurity radiation fraction of $80 \%$ was studied. In the large machine size DEMO, the core density tends to decrease compared with the small size DEMO, such as SlimCS. Therefore, the low SOL density case, i.e., no fuel gas puff for the peripheral plasma cooling, was investigated in this study. In the SONIC simulation, the low SOL density less than $1.4 \times 10^{19} \mathrm{~m}^{-3}$ was obtained. Even in such a low SOL density, the partial detachment was obtained at the outer divertor, due to the large impurity radiation in the SOL and divertor region. The peak of the target heat load was $7 \mathrm{MW} / \mathrm{m}^{2}$, which can be handled by the tungsten monoblock target with the $\mathrm{Cu}$-alloy cooling tube. However, the peak of the ion temperature at the target was considerably high, which causes significant erosion of the target. The divertor power handling and decrease in the ion temperature have to be proceeded by development of the scenario of the divertor plasma operation as well as the core plasma design and the engineering design.

In the engineering study, design of the tungsten monoblock target with the water cooling and the $\mathrm{Cu}$-alloy cooling tube have been progressed. The MCNP-5 neutronics analysis showed the DPA rate of 1-2 DPA for a full power year at the $\mathrm{Cu}$-alloy tube for the $8 \mathrm{~m}$-sized DEMO with the fusion power of $1.5 \mathrm{GW}$. Therefore, the $\mathrm{Cu}$-alloy cooling pipe can be applied for the divertor unit on the high heat flux region, while the replacement of the $\mathrm{Cu}$ alloy cooling unit is expected every $1-2$ years. The divertor cassette with two different cooling route, i.e., the RAFM pipe and $\mathrm{Cu}$-alloy pipe, was designed and the peak target heat load of $10 \mathrm{MW} / \mathrm{m}^{2}$ can be removed. The heat transport analysis for the tungsten monoblock with the $\mathrm{Cu}$ alloy pipe showed the maxium temperature of $1021^{\circ} \mathrm{C}$ at the tungsten surface and $331^{\circ} \mathrm{C}$ at the $\mathrm{Cu}$-alloy pipe. In these temperature range, the above monoblock concept can be acceptable.

\section{Acknowledgments}

This work is mainly carried out within the framework of the DEMO Design Activity under the the Broader Approach collaboration between Euratom and Japan and the Joint Special Design Team for fusion DEMO, and partially supported by Grants-in-Aid for Scientific Research of Japan Society for the Promotion of Science. SONIC simulations were carried out using the HELIOS supercomputer system at International Fusion Energy Research Centre, Aomori, Japan, under the Broader Approach.

[1] K. Tobita et al., Nucl. Fusion 47, 892 (2007).

[2] K. Tobita et al., Nucl. Fusion 49, 075029 (2009).

[3] H. Kawashima et al., Nucl. Fusion 49, 065007 (2009).

[4] N. Asakura et al., J. Plasma Fusion Res. SERIES 9, 136 (2010).

[5] N. Asakura et al., Nucl. Fusion 53, 123013 (2013).

[6] K. Hoshino et al., Contrib. Plasma Phys. 52, 550 (2012).

[7] N. Asakura et al., Fusion Sci. Technol. 63, No.1T, 70 (2013).

[8] Y. Sakamoto et al., 25th IAEA Fusion Energy Conference, FIP/3-4Rb, St Petersburg, Russian Federation, 2014.

[9] K. Kawashima et al., Plasma Fusion Res. 1, 031 (2006).

[10] K. Shimizu et al., Nucl. Fusion 49, 065028 (2009).

[11] K. Hoshino et al., J. Nucl. Mater. 463, 573 (2015).

[12] K. Hoshino et al., Contrib. Plasma Phys. 56, 657 (2016).

[13] S. Tokunaga et al., the 22th International Conference on Plasma Surface Interactions in Controlled Fusion Devices, P.3.105, Rome, Italy, 2016.

[14] K. Hoshino et al., 25th IAEA Fusion Energy Conference, FIP/P8-11, St Petersburg, Russian Federation, 2014.

[15] H.D. Pacher et al., J. Nucl. Mater. 415, S492 (2011).

[16] A.S. Kukushkin et al., J. Nucl. Mater. 438, S203 (2013).

[17] K. Hoshino et al., Contrib. Plasma Phys. 54, 404 (2014).

[18] S.A. Fabritsiev et al., J. Nucl. Mater. 233-237, 127 (1996).

[19] G.M. Kalinin et al., J. Nucl. Mater. 307-311, 668 (2002). 